\title{
Detecting transits from Earth-sized planets around Sun-like stars
}

\author{
S. Carpano ${ }^{1}$ and M. Fridlund ${ }^{2}$
}

\author{
1 XMM-Newton Science Operations Centre, ESAC, ESA, PO Box 50727, 28080 Madrid, Spain \\ e-mail: scarpano@sciops.esa.int \\ 2 ESA Astrophysics Missions Division, Research and Scientific Support Department, ESTEC, PO Box 299, 2200 AG, Noordwijk, \\ The Netherlands
}

Received 18 December 2007 / Accepted 25 March 2008

\section{ABSTRACT}

\begin{abstract}
Context. Detecting regular dips in the light curve of a star is an easy way to detect the presence of an orbiting planet. CoRoT is a Franco-European mission launched at the end of 2006, and one of its main objectives is to detect planetary systems using the transit method.

Aims. In this paper, we present a new method for transit detection and determine the smallest detected planetary radius, assuming a parent star like the Sun.

Methods. We simulated light curves with Poisson noise and stellar variability, for which data from the VIRGO/PMO6 instrument on board SoHO were used. Transits were simulated using the Universal Transit Modeller software. Light curves were denoised by the mean of a low-pass and a high-pass filter. The detection of periodic transits works on light curves folded at several trial periods with the particularity that no rebinning is performed after the folding. The best fit was obtain when all transits are overlayed, i.e. when the data are folded at the right period.

Results. Assuming a single data set lasting $150 \mathrm{~d}$, transits from a planet with a radius down to $2 R_{\oplus}$ can be detected. The efficiency depends neither on the transit duration nor on the number of transits observed. Furthermore we simulated transits with periods close to $150 \mathrm{~d}$ in data sets containing three observations of $150 \mathrm{~d}$, separated by regular gaps with the same length. Again, planets with a radius down to $2 R_{\oplus}$ can be detected.

Conclusions. Within the given range of parameters, the detection efficiency depends slightly on the apparent magnitude of the star but neither on the transit duration nor the number of transits. Furthermore, multiple observations might represent a solution for the CoRoT mission for detecting small planets when the orbital period is much longer than the duration of a single observation.
\end{abstract}

Key words. stars: planetary systems - techniques: photometric - methods: data analysis - occultations

\section{Introduction}

Beginning with the discovery by Mayor \& Queloz (1995) 12 years ago of a giant planet, $51 \mathrm{Peg}$ b, around its Sun-like host star, the search for planets outside the solar system has become a major topic in astrophysics. Among the various methods available to search for exoplanets, the transit method presents the opportunity of directly determining the planet's radius relative to that of its parent star and, if more than one transit is observed, its orbital period. It also has the advantage of beeing possible to monitor many thousands of target stars simultaneously. This is of course necessary, since the probability that a planet transits its star depends on the radii of star and planet, as well as the orbital distance and the eccentricity (Rauer \& Eriksson 2007). It is never a large number, viz.

Probability $=0.0045\left(\frac{1 \mathrm{AU}}{a}\right) \times\left(\frac{r_{\mathrm{s}}+r_{\mathrm{p}}}{1_{\odot}}\right) \times\left[\frac{1+e \cos \left(\frac{\omega}{2}\right)}{1-e^{2}}\right]$,

where $a$ is the semi major axis of the planetary orbit, $r_{\mathrm{s}}$ and $r_{\mathrm{p}}$ are the stellar and planetary radii respectively, $e$ the eccentricity, and $\omega$ the longitude of the periastron. It is easy to see that, for an Earth analogue, the probability of a transit beeing observed is about $0.5 \%$, while for a similar planet at $0.05 \mathrm{AU}$, the geometrical probability rises to about $10 \%$. When also folding in the poorly known factor $\eta_{\text {Planet }}$ (number of planets per star system), one realizes this need for (simultaneous) observations of large numbers of stars.

Diminishing of the light curve relative to the light from the star, the photometric signal $P$, is of course immediately translatable into the ratio of planetary to stellar radius

$P=\frac{\Delta S}{S}=\left[\frac{R_{\text {Planet }}}{R_{\text {Star }}}\right]^{2}$.

Therefore, the minimum size of the planet that can be detected will only depend on the precision with which one can measure the photometric output from the star and know the stellar radius.

Successful results have been obtained for a score of transiting objects, since the first detection by Charbonneau et al. (2000), but all of these are essentially Jupiter-like objects in very close orbits (i.e. less than a few days). If we want to detect and study planets more akin to our own Earth, we are facing formidable problems, where the stellar radius currently can only be discerned from model calculations, and even then is still contains severe uncertainties. The achievable photometric precision is governed by the problem of acquiring long and un-interupted sequences of data. When observing from the ground we are faced with enormous challenges. In contrast, by launching a telescope into space, into an orbit allowing these long, uninterrupted data acquisitions, much higher precision can be achieved. Nevertheless, by improving the photometric signal, one also increases the ambition. Thus, with the advent of 
space-based assets, the goal is now set at the actual detection of a planet with the same radii as our own Earth.

Simultaneously, during the development of the first space missions, a number of transit-detection algorithms have been proposed in the literature including Bayesian algorithms (i.e., Aigrain \& Favata 2002), matched filters (i.e., Jenkins et al. 1996), box-shape transit finder (Aigrain \& Irwin 2004), and the box-fitting least squares method (Kovács et al. 2002). A comparison of the capabilities of existing methods was first made by (Tingley 2003a; revised in Tingley 2003b) and later by Moutou et al. (2005) on updated versions of the algorithms. Besides the detection method, filtering of photon noise and stellar variability also play an important role. In Carpano et al. (2003), we used an optimal filter to significantly reduce the stellar variability and enhance the $\mathrm{S} / \mathrm{N}$ ratio of the transits. However, this filter uses a reference signal and hence some a priori information of the shape of the signal we are looking for.

The CoRoT spacecraft was launched on December 27, 2006 and has been carrying out routine scientific operations since February 2, 2007. CoRoT stands for COnvection, ROtation , and planetary Transits. Briefly, this satellite is carrying out ultra high-precision photometry for the dual purpose of studying asteroseismological signals from stars and both discovering and studying extra-solar planets with unprecedented precision. The focal plane is divided into two segments, one each for these objectives. Each of the parts of the focal planet (designated "exo" and "seismo") is equipped with two CCD-based detectors. The exoplanetary detector records the light curve of up to 12000 targets. Of these, $20 \%$ have a time sampling of $32 \mathrm{~s}$, while the rest are sampled every $512 \mathrm{~s}$. Continuous monitoring of up to 150 days for each selected field is carried out, and a grism in front of the detector allows thousands of the light curves to be obtained in three colours for stars with visual magnitudes from about 12 to 16 . For further basic information about the mission we refer to ESA SP-1306 (2006, The CoRoT Mission) for a description of all aspects as of pre-launch.

One of the main goals of CoRoT is to detect smaller exoplanets than previously possible with any method. Radial velocity measurements is currently limited to the detection of planets 5-10 times as massive as the Earth itself in orbits around very low-mass stars (Udry et al. 2007). The occultation method as carried out from the ground also has limitations imposed by both the atmosphere and, more important, by the interruptions inherent in any lightcurve obtained from the surface, although extensive networks developed in recent years alleviate this. In this case, the depth in the light curve that can be obtained from the ground under the best of circumstances is at best a few tenths of a percent (Rauer \& Eriksson 2007), representative of planets of either the Jupiter or Saturn class. In the case of CoRoT, on the other hand, it has been shown by in-orbit verification that the data is photon-noise-limited, or almost so, in the whole magnitude range for the exo-planetary segment for integration times shorter than $1 \mathrm{~h}$ (Baglin et al 2007). The detections of Earthsized planets then becomes possible with the CoRoT mission - at least for shorter orbital periods - but is nevertheless is a challenging task.

In this paper we present a new, although simple, transit detection method that could be applied to CoRoT data after having applied a smoothing and detreding filter. We do not attempt to compare the efficiency of the method with respect to other ones, for which a proper analysis should be performed much like the one in Tingley (2003a) and Moutou et al. (2005). Here we concentrate on describing the method, and quote its capabilities and its efficiency at detecting small planets when few transits are

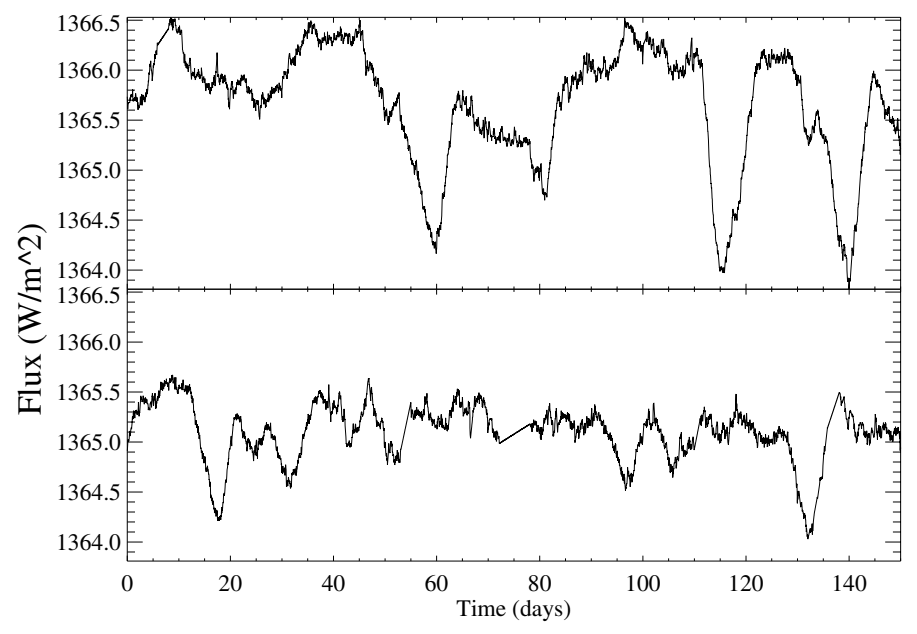

Fig. 1. VIRGO/PMO6 data from the SoHO satellite, from year 2000 (top) and 2006 (bottom) used to simulate stellar activity at high and low level, respectively.

observed. The detection is applied to simulated light curves with Sun-like stellar variability, lasting 150 days as is the case for CoRoT. Assuming the radius of the parent star is fixed to solar value, we check the radius the planet needs for beeing detected. Stellar apparent magnitude, transit duration and orbital period, hence the number of transits, are all taken to be random in a given range of values. To avoid a long computation time, we limited our simulation to 100 light curves for each stellar activity level and therefore did not follow the approach of Tingley (2003a), which uses Monte Carlo simulations.

We also tested the transit detection method in the case of three observations of the same object separated by 150 days, where only one transit is visible in each data set. This simulates the observation of the same region at several separated epochs.

The paper is organised as follows. Section 2 describes the way the simulated input light curves were derived. In Sect. 3 we describe the filtering and the transit detection method we have applied. The performances of the method are evaluated in Sects. 4 and 5, for the single and multiple observations, respectively. Finally, the discussion of our results is presented in Sect. 6.

\section{Data simulations}

To test the efficiency of our transit detection method in finding Earth-sized planets around a Sun-like star, we simulated light curves using VIRGO/PMO6 data (Fröhlich et al. 1997) from the SOHO telescope as done in Carpano et al. (2003). Since data from VIRGO are only available on a hourly time grid, we interpolated them to have a data sampling of $10 \mathrm{~min}$. The total duration of the light curve is $150 \mathrm{~d}$ as expected from the CoRoT mission (where the time resolution is $32 \mathrm{~s}$ or $512 \mathrm{~s}$ ). To simulate a high level of stellar activity, VIRGO data from the year 2000 were used, while low activity was represented by data from the year 2006. The corresponding VIRGO/PMO6 data sets are shown in Fig. 1. On the top of these data, we added Poisson noise, assuming that $\Delta F / F=7 \times 10^{-4}$, where $F$ is the total number of photons collected in $1 \mathrm{~h}$ for a star of apparent magnitude 15.5. This is what is expected for the CoRoT mission. No instrumental noise of any kind has been introduced into the simulated light curves.

Dips associated with the transit of a planet are simulated using the Universal Transit Modeller (UTM) software, 
Table 1. Parameters used in the Universal Transit Modeller software to simulate the transit of a planet in front of its parent star.

\begin{tabular}{ll}
\hline \hline Parameter & Value/range of value \\
\hline Star mass, $M_{\star}$ & $1 M_{\odot}$ (fixed) \\
Star radius, $R_{\star}$ & $1 R_{\odot}$ (fixed) \\
Limb darkening coefficient, $L_{\mathrm{d}}$ & 0.51 (fixed) \\
Apparent magnitude, $V$ & {$[12-16] \mathrm{mag}$} \\
Transit duration, $t_{\mathrm{dur}}$ & {$[4.5-36] \mathrm{h}$} \\
Planet radius, $R_{\mathrm{pl}}$ & {$[1-5] R_{\oplus}$} \\
Orbital period, $P$ & {$[25-50] \mathrm{d}$} \\
Orbital phase, $P h$ & {$[0-1]$} \\
\hline
\end{tabular}

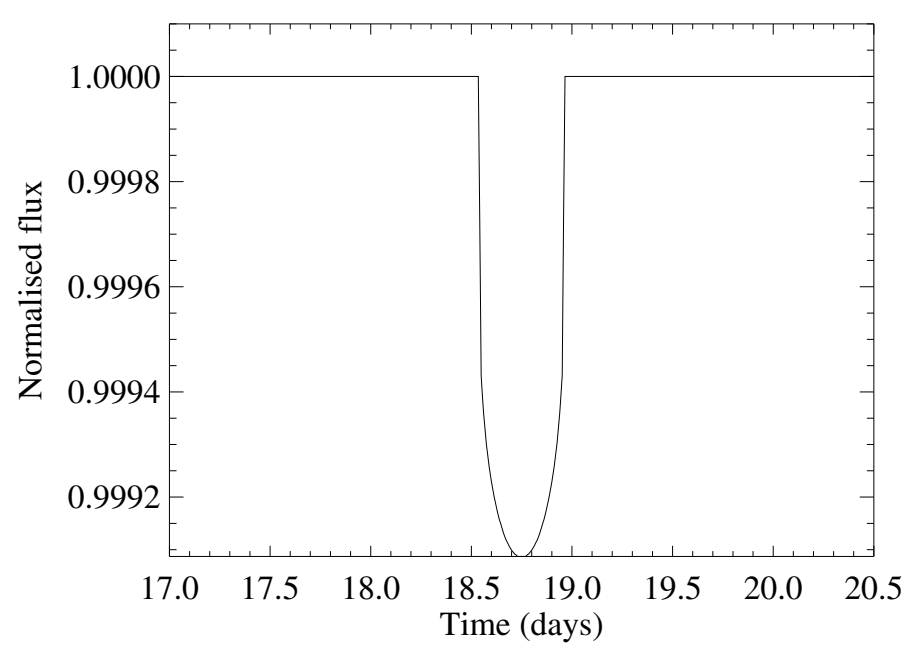

Fig. 2. Output of the UTM code, zoomed around the first transit, where $R_{\mathrm{pl}}=3 R_{\oplus}, P=37.5 \mathrm{~d}, P h=0.5, t_{\mathrm{dur}}=10 \mathrm{~h}$.

developed by Deeg (1999). The utilised parameters values are listed in Table 1: the mass, $M_{\star}$, the radius, $R_{\star}$, and limbdarkening coefficient, $L_{\mathrm{d}}$, of the parent star, which are kept fixed to solar values. The radius of the planet, $R_{\mathrm{pl}}$, the orbital period, $P$, and the phase, $p h$, (thus the number of transits), and the transit duration change randomly within a given range of values. We require that at least 3 transits are present in the light curves, with a maximum of 6 transits. The apparent magnitude, ranging from 12 to 16 is related to the Poisson noise added to the light curve. An example of the output of the UTM code, zoomed around the first transit, is shown in Fig. 2 where $R_{\mathrm{pl}}=3 R_{\oplus}$, $P=37.5 \mathrm{~d}, P h=0.5, t_{\text {dur }}=10 \mathrm{~h}$. The sharp drop and increase in flux are caused by the ingress/egress of the planet, while the smoother variation close to the bottom is due to limb-darkening effects.

\section{Filtering and periodic transit detection}

\subsection{Filtering}

To filter the Poisson noise and detrend the light curve for stellar variability, we used a low-pass and a high-pass filter, respectively, equivalent to a standard box-shaped filter in the Fourier domain. The disadvantage of these filters is that the shape of the dip is slightly changing. Without passing through the Fourier domain, the data sets are filtered by a boxcar smooth over a certain width, using the IDL tool smooth. To reduce the Poisson noise, we smooth the data over a width of 25 (in units of $10 \mathrm{~min}$ ). This width, linked to our data sampling, limits the lower value of our simulated transit duration $(4.5 \mathrm{~h})$. Outputs from the CoRoT mission have a data sampling of $32 \mathrm{~s}$ (in the oversampled mode), therefore decreasing the lower limit for the transit duration to be

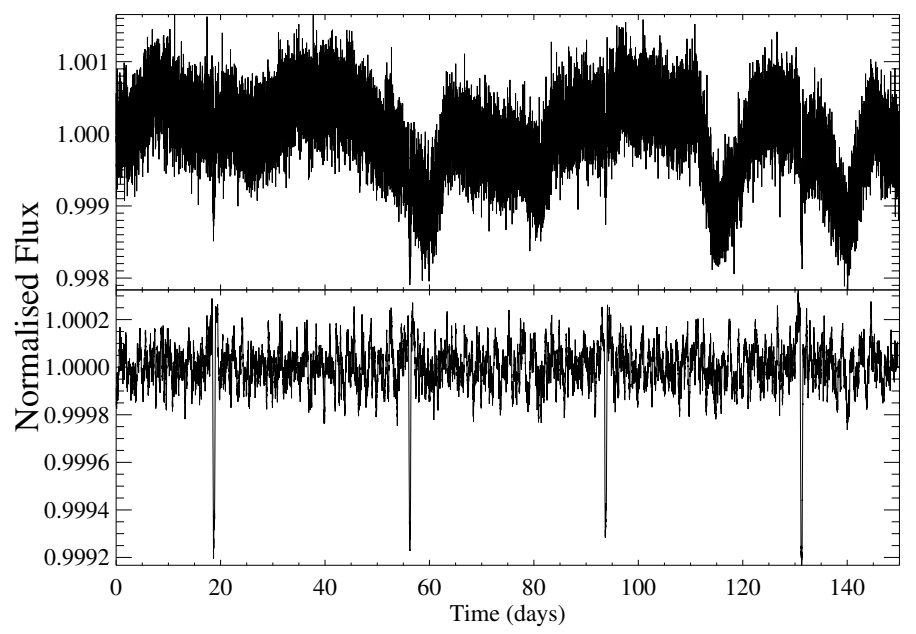

Fig. 3. Simulated light curve before and after filtering. Transits are built with the same parameters as in Fig. 2, stellar variability is taken at the high level, and $V=12 \mathrm{mag}$.

detected. The smoothing unfortunately reduces the sharpness of the transit ingress/egress. This shape's characteristic can be used to distinguish dips from an eclipse with respect to dips from stellar variability; however, this will have no effect on our detection method as described below.

The detrending is performed in two steps: we first smooth the data over a large width (wider than the maximal transit duration, i.e. $1.5 \times t_{\text {dur max }}=54 \mathrm{~h}$ ) and then remove the long-term smoothed light curve to the previous short-term smoothed one. This results in a light curve filtered for stellar variability and Poisson noise. Extreme values are set to the mean light curve value (1 if normalised). An example of a light curve before and after filtering is given in Fig. 3, where the parameters for the UTM code are taken as in Fig. 2. Stellar variability is assumed to be high, while the apparent magnitude $V=12 \mathrm{mag}$.

\subsection{Periodic transit detection method}

Our transit detection method has the advantage of beeing very simple. We first fold the light curve over a given range of trial periods. The only difference with respect to the conventional epoch-folding method (Leahy et al. 1983) is that the data are not rebinned after folding. On the folded data set, for each trial period, we first sort the data in an ascending order for the phase values and then fit a Gaussian function using the IDL function gaussfit. Results are shown in Fig. 4: when data are folded at the correct period, the Gaussian fits the data relatively well (top of Fig. 4). In contrast when data are not folded at the correct period, the width of the Gaussian function is constrained by the data sampling, which results in a bad fit. This is also the case when folding at half of the period, one third of it, etc. Testing over a full range of trial periods, the best value is given by the minima of the chi-square function as shown in the next section, in Fig. 5. Note that the fit is performed over the entire folded light curve, while in Fig. 4 only the region around the transit dip is shown. The shape of the transit is modified by the filter, which creates bumps before and after it.

Other minima can be observed at multiple values of the correct period. If the $\mathrm{S} / \mathrm{N}$ ratio is not too low or if the period step value is not too high, the chi-square value associated with these sub-harmonics will always be at the secondary minima. No signal will be found at half of the period, one third of it, etc., and 


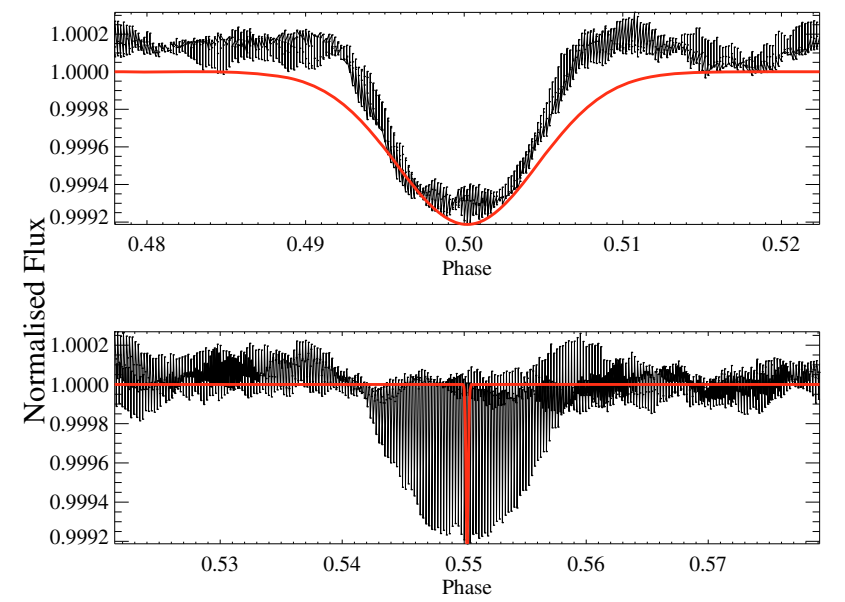

Fig. 4. Light curve folded at the correct (top) and wrong (bottom) period, without any rebinning. Overlayed is the best fit Gaussian function (see text for more details).

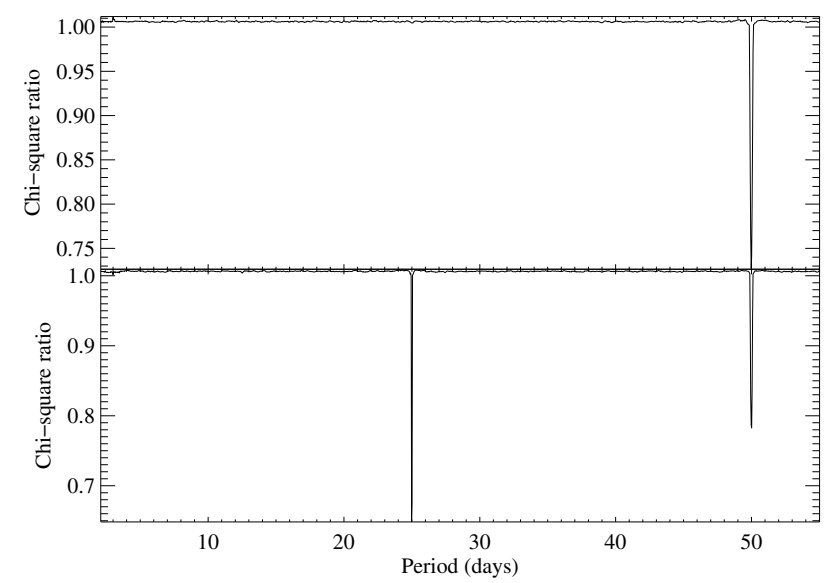

Fig. 5. Chi-square ratio values for the Gaussian fit of light curves folded over trial periods ranging from $5 \mathrm{~h}-55 \mathrm{~d}$, for an input period at $50 \mathrm{~d}$ (top) and $25 \mathrm{~d}$ (bottom). The minimum of the curve gives the value for the best-fitted period.

thus no harmonics are present in the periodogram. The period step should be chosen as small as possible, ideally one time unit, or at least $1 / n$th of the transit duration when no more than $n$ transits are expected.

This detection method presents the disadvantage that the position of the fitted Gaussian function is given by the minima of the filtered light curve. If this minima is not associated with any of the transits but with some dip coming from the stellar activity, for example, the correct period will not be found.

Another property of the method is that the detection efficiency does not depend on the number of transits observed. The right period of a data set containing a large number of transits will be detected in the same way that with only a few observed occultations. However, the presence of many transits will increase the confidence level of the period detection (see next section).

Comparing with other methods qualitatively, this one has the advantage of not needing to create a set of test light curves to be compared to the observed one as done for the matchedfilter (Jenkins et al. 1996) or the method of Protopapas et al. (2005) (which uses analytical function to characterise a transit). It does not need any assumption on any parameter, like the transit duration, as with the sliding transit template correlation

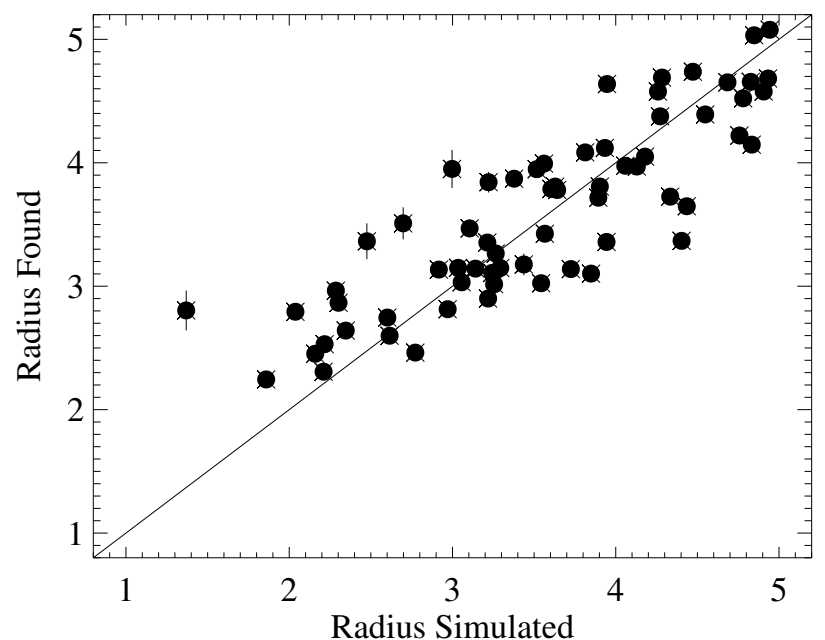

Fig. 6. Correlation between the simulated planet's radius and the one determined by the Gaussian fit, when the right period has been recovered.

developed by Team 1 in Moutou et al. (2005). The nonimprovement of period detection with an increase in the number of transits is also observed in the method used by Team 2 in Moutou et al. (2005). They make a box search for all data points deviating from the average signal by 3 sigma, remove spurious events, and subsequently search for a periodicity in all detected epochs. Our method has the advantage of doing everything at once. Furthermore, we do not detect harmonics of the periodic signal but only the sub-harmonics, which decreases the noise level in the chi-square function. Of course a proper comparison would be necessary to test the efficiency of the periodic transit detection and/or time consumption of this method with respect to existing ones.

\subsection{Confidence levels}

To determine the confidence level of a period detection, we estimated the ratio between the chi-square resulting from a linear fit and the one resulting from a Gaussian fit. We call this the $\chi_{\text {ratio }}^{2}$ (see Fig. 5, top, for three transits, and bottom, for six transits). The second chi-square increases with the number of transits, but the first increases faster. Thus when increasing the number of transits, the $\chi_{\text {ratio }}^{2}$ function goes to lower minima, therefore enhancing the confidence level. We then compared this minimum to that expected for transit free data. In practice, we simulated 100 light curves without transits using solar data both at high and low activity levels. We filter the data, search for a periodic signal transit, and calculate the $\chi_{\text {ratio }}^{2}$. It turns out that $68 \%$ of the light curves will provide a $\chi_{\text {rati }}^{2}>0.971$, while $90 \%$ will be $>0.989$ and $99 \%$ greater than 0.997 . Thus a transit is detected at a confidence level of $90 \%$ if the $\chi_{\text {ratio }}^{2}>0.989$. These thresholds do not depend on the activity level of the star since they have been pre-filtered.

\subsection{Estimate of the planetary radius}

Since we kept the radius of the parent star equal to $1 R_{\odot}$, the depth of the transit will directly estimate of the planet radius. This depth is given by the amplitude of the fitted Gaussian function. In Fig. 6 we show the correlation between the simulated planet's radius and the one determined by the Gaussian fit, when 
the right period has been recovered. The maximal deviation between the simulated and detected radius is $\sim 1 R_{\oplus}$.

We also tried to get an estimate of the transit duration, from the width of the fitted Gaussian function; but due to the high level of noise, no correlation was found for these two parameters. However, in a second step once the transits have been detected, a deeper analysis of the source allows a more accurate fit. An estimation of the transit duration is then provided by the width $(2 \times 4$ sigma $)$ of the Gaussian function.

\subsection{How the method deals with unevenly sampled data or with short/long data gaps}

The detection method described above has the advantage of not being too affected by unevenly distributed data. The prior filtering of the light curve, on the other hand, works properly only for evenly sampled data. This problem can be solved easily by rebinning the uneven data set on to a regularly-spaced grid. Concerning the gaps, if short ones are present (short with respect to the transit duration), this will again affect the results only in the pre-filter part. A solution will then consist in interpolating the data over the missing values. If medium-sized gaps are present, the procedure and results will stay the same as long as no transits are present in the gaps. If transits are present in these gaps, the correct period will not be found even if the dips are very pronounced. When long-lasting gaps are present, each data set should then be pre-filtered separately. The detection method works also well with long gaps, depending on their length and the orbital period to be detected. This is of course important if we want to search for planets with periods longer than $\approx 1 / 3$ of the length of the uninterrupted data, and thus have to return to observing the same object after an interruption that could be up to the same length as the original data set (e.g. because of reasons of orbital mechanics with respect to the spacecraft). An application of this case is given in Sect. 5 .

\section{Performances of the detection method}

To test the efficiency of the detection method, we simulated 100 light curves as described in Sect. 2 (Table 1). Results are displayed in Fig. 7 for the case of high stellar activity and in Fig. 8 when stellar activity is low. We compare the minimum size of the detected planet as a function of the apparent magnitude (top), the transit duration (middle), and the orbital period (bottom). Table 2 shows the total number of simulations (NB., 1 st column) for a given range of the input parameters of Table 1: radius of the planet, magnitude, transit duration, and period (related to the number of transits), for high stellar activity level. The 2nd, 3rd, 4th, and 5th columns show, respectively, the number (in \%) of correct period detection (YES), of failed period detection (NO), of false alarm (F.A.), and of missed detection (M.D.). Table 3 shows the same results but for a low activity level.

The main results are that, when assuming solar variability and Poisson noise level as given in Sect. 2, planets with a radius down to $\sim 2 R_{\oplus}$ can be recovered when the parent star has a radius of $1 R_{\odot}$. The performance depends slightly on the apparent magnitude but not on the transit duration. Finally, as expected, the efficiency does not depend on the orbital period, which is directly correlated to the number of transits.

\section{Multiple observations with long and regular gaps}

In this section, we investigate one possible application for the CoRoT mission: what if a planet has an orbital period close to
Table 2. Performance of the periodic transit detection algorithm in case of high stellar activity.

\begin{tabular}{|c|c|c|c|c|c|}
\hline \multicolumn{6}{|c|}{ Planet radius $\left(R_{\odot}\right)$} \\
\hline Radius & NB. & YES (\%) & $\mathrm{NO}(\%)$ & F.A. $(\%)$ & M.D. (\%) \\
\hline $1-2$ & 22 & 23 & 77 & 9 & 13 \\
\hline $2-3$ & 27 & 48 & 52 & 22 & 0 \\
\hline $3-4$ & 31 & 94 & 6 & 3 & 10 \\
\hline $4-5$ & 20 & 100 & 0 & 0 & 0 \\
\hline \multicolumn{6}{|c|}{ Magnitude (Mag) } \\
\hline Mag. & NB. & YES $(\%)$ & $\mathrm{NO}(\%)$ & F.A. $(\%)$ & M.D. (\%) \\
\hline $12-13$ & 26 & 81 & 19 & 0 & 4 \\
\hline $13-14$ & 24 & 79 & 21 & 4 & 4 \\
\hline $14-15$ & 25 & 72 & 28 & 8 & 8 \\
\hline $15-16$ & 25 & 36 & 64 & 24 & 8 \\
\hline \multicolumn{6}{|c|}{ Transit duration (hours) } \\
\hline Tdur & NB. & YES $(\%)$ & $\mathrm{NO}(\%)$ & F.A. $(\%)$ & M.D. (\%) \\
\hline $4-12$ & 22 & 77 & 23 & 9 & 0 \\
\hline $12-20$ & 28 & 68 & 32 & 11 & 7 \\
\hline $20-28$ & 26 & 65 & 35 & 8 & 8 \\
\hline $28-36$ & 24 & 58 & 42 & 8 & 8 \\
\hline \multicolumn{6}{|c|}{ Period (days) } \\
\hline $\mathrm{P}$ & NB. & YES $(\%)$ & NO $(\%)$ & F.A. $(\%)$ & M.D. (\%) \\
\hline $24-30.5$ & 28 & 75 & 25 & 11 & 7 \\
\hline $30.5-37$ & 26 & 62 & 38 & 12 & 4 \\
\hline $37-43.5$ & 25 & 68 & 32 & 8 & 4 \\
\hline $43.5-50$ & 21 & 62 & 38 & 5 & 10 \\
\hline
\end{tabular}

Table 3. Same as Table 2 for low stellar activity level.

\begin{tabular}{|c|c|c|c|c|c|}
\hline \multicolumn{6}{|c|}{ Planet radius $\left(R_{\odot}\right)$} \\
\hline Radius & NB. & YES $(\%)$ & $\mathrm{NO}(\%)$ & F.A. $(\%)$ & M.D. (\%) \\
\hline $1-2$ & 24 & 8 & 92 & 8 & 4 \\
\hline $2-3$ & 25 & 60 & 40 & 12 & 12 \\
\hline $3-4$ & 30 & 93 & 7 & 0 & 0 \\
\hline $4-5$ & 21 & 100 & 0 & 0 & 5 \\
\hline \multicolumn{6}{|c|}{ Magnitude (Mag) } \\
\hline Mag. & NB. & YES $(\%)$ & $\mathrm{NO}(\%)$ & F.A. $(\%)$ & M.D. (\%) \\
\hline $12-13$ & 32 & 75 & 25 & 0 & 0 \\
\hline $13-14$ & 26 & 65 & 35 & 4 & 4 \\
\hline $14-15$ & 20 & 80 & 20 & 0 & 15 \\
\hline $15-16$ & 22 & 41 & 59 & 18 & 5 \\
\hline \multicolumn{6}{|c|}{ Transit duration (hours) } \\
\hline Tdur & NB. & YES (\%) & NO (\%) & F.A. (\%) & M.D. (\%) \\
\hline $4-12$ & 21 & 71 & 29 & 5 & 10 \\
\hline $12-20$ & 27 & 78 & 22 & 0 & 7 \\
\hline $20-28$ & 25 & 56 & 44 & 8 & 0 \\
\hline $28-36$ & 27 & 59 & 41 & 7 & 4 \\
\hline \multicolumn{6}{|c|}{ Period (days) } \\
\hline $\mathrm{P}$ & NB. & YES $(\%)$ & NO $(\%)$ & F.A. $(\%)$ & M.D. (\%) \\
\hline $24-30.5$ & 27 & 59 & 41 & 7 & 7 \\
\hline $30.5-37$ & 30 & 60 & 40 & 7 & 7 \\
\hline $37-43.5$ & 24 & 67 & 33 & 4 & 0 \\
\hline $43.5-50$ & 19 & 84 & 16 & 0 & 5 \\
\hline
\end{tabular}

$1 \mathrm{yr}$, when a transit is observed in the first $150 \mathrm{~d}$ data set. Since the satellite cannot observe the same field again before another $150 \mathrm{~d}$, would it still be possible to recover the orbital period of the planet? Since the detection method finds the right period only when all visible transits are overlayed, the answer is yes. Again, as in the case of a single observation, a requirement for the period detection is that the minimum of the global light curve is associated with a transit.

We simulated three $150 \mathrm{~d}$ observations separated by gaps of the same length. The global light curve then lasts for about $2.5 \mathrm{yr}$ (see Fig. 9 before and after filtering). To ensure that at least one 

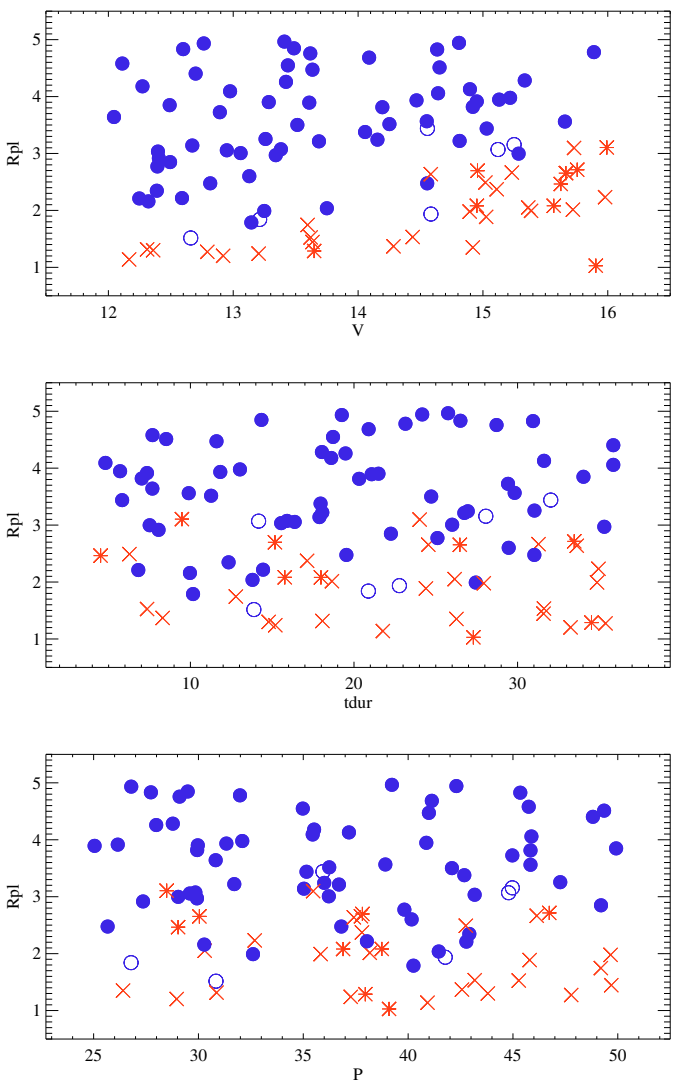

Fig. 7. Results of searching for small planets in 100 lightcurves. Performance of the periodic transit detection algorithm in case of high stellar activity. Dots are associated with a successful recovery of the period with a confidence level $>90 \%$ and circles with a confidence level $<90 \%$. A failed recovery of the simulated period is shown by a cross when the confidence level is $<90 \%$ and by a star for a level $>90 \%$. Parameters and units are explained in Sect. 2.

Table 4. Same as Table 2 but in the case of three $150 \mathrm{~d}$ observations separated by gaps of $150 \mathrm{~d}$.

\begin{tabular}{cccccc}
\hline \hline \multicolumn{6}{c}{ Planet radius $\left(R_{\odot}\right)$} \\
Radius & NB. & YES $(\%)$ & NO $(\%)$ & F.A. $(\%)$ & M.D. $(\%)$ \\
\hline $1-2$ & 23 & 9 & 91 & 4 & 0 \\
$2-3$ & 23 & 65 & 35 & 4 & 4 \\
$3-4$ & 29 & 83 & 17 & 7 & 0 \\
$4-5$ & 25 & 96 & 4 & 0 & 0 \\
\hline \multicolumn{6}{c}{ Magnitude (Mag) } \\
Mag. & NB. & YES (\%) & NO (\%) & F.A. (\%) & M.D. (\%) \\
\hline $12-13$ & 28 & 79 & 21 & 0 & 0 \\
$13-14$ & 24 & 79 & 21 & 0 & 4 \\
$14-15$ & 23 & 65 & 35 & 4 & 0 \\
$15-16$ & 25 & 36 & 64 & 12 & 0 \\
\hline \multicolumn{7}{c}{ Transit duration (hours) } \\
Tdur & NB. & YES (\%) & NO (\%) & F.A. (\%) & M.D. (\%) \\
\hline $4-12$ & 32 & 69 & 31 & 3 & 0 \\
$12-20$ & 31 & 65 & 35 & 0 & 0 \\
$20-28$ & 18 & 50 & 50 & 11 & 0 \\
$28-36$ & 19 & 74 & 26 & 5 & 5 \\
\hline \multicolumn{7}{c}{}
\end{tabular}

transit is visible in each data set, we set the first one at the half of the first observation and allowed the period to change over a low value $P=300 \mathrm{~d} \pm 37.5 \mathrm{~d}$. In this exercice we only focused on recover periods close to $150 \mathrm{~d}$.

Figure 10 and Table 4, show the same results as Fig. 7 and Table 2, respectively, but for multiple observations. The main
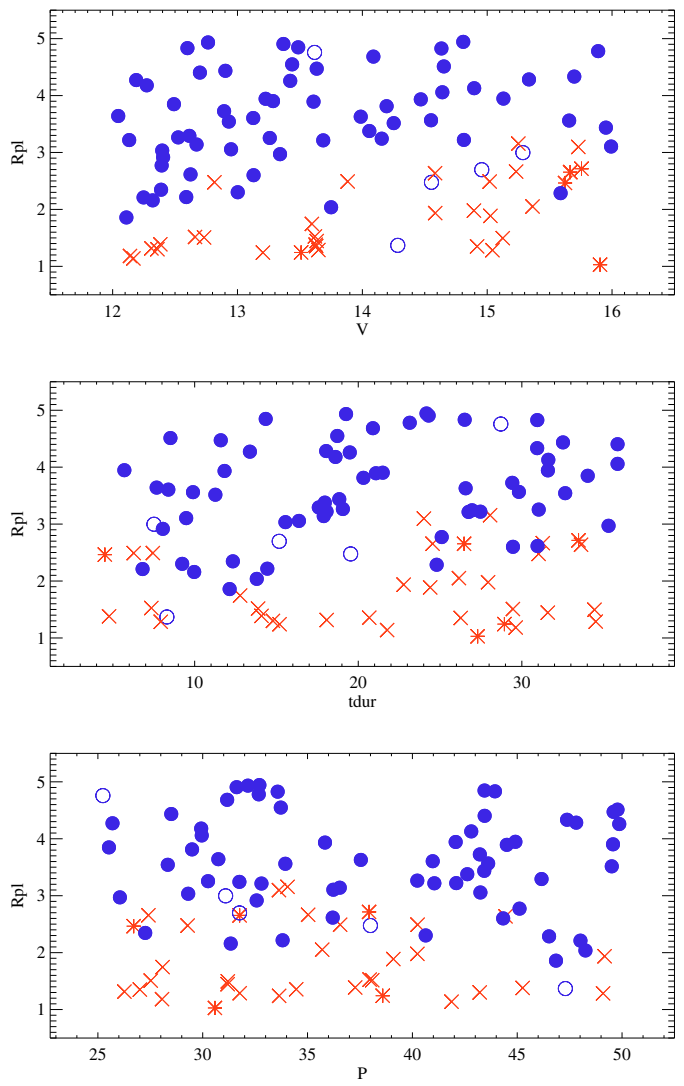

Fig. 8. Same as Fig. 7 but for low stellar activity.

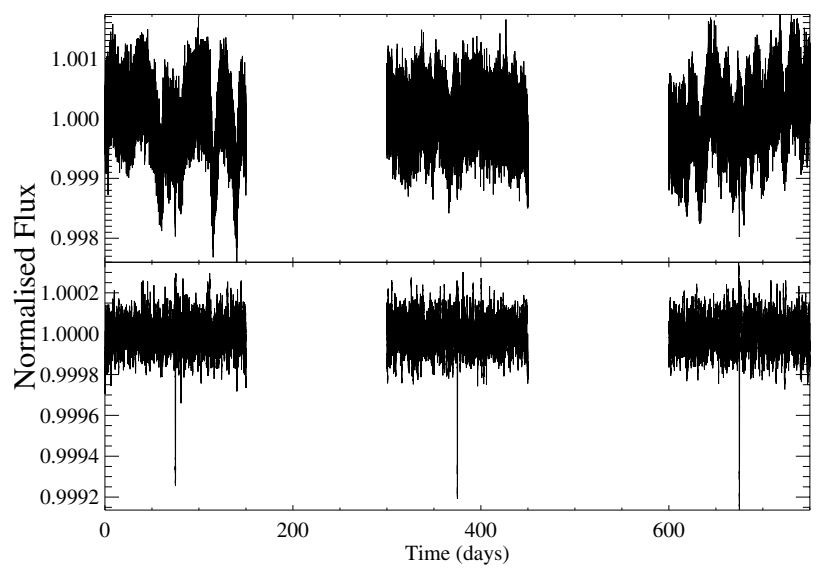

Fig. 9. Same as Fig. 3 but in the case of three $150 \mathrm{~d}$ observations separated by gaps of $150 \mathrm{~d}$. The phase of the first transit is at the half of the first data set.

conclusion from Fig. 10 is that regular longlasting gaps do not affect the recovery of the transit period when close to $150 \mathrm{~d}$. Results are similar to the case of one single data set with low stellar activity. The threshold for the minimum detected planet radius is again around $2 R_{\oplus}$.

\section{Summary}

In this paper, we have presented a new method for detecting transits of a planet orbiting around its parent star, and we investigate the limit of the smallest detectable planet when assuming that the star has similar characteristics to the Sun. We simulated light curves as described in Sect. 2, and, before applying the transit 

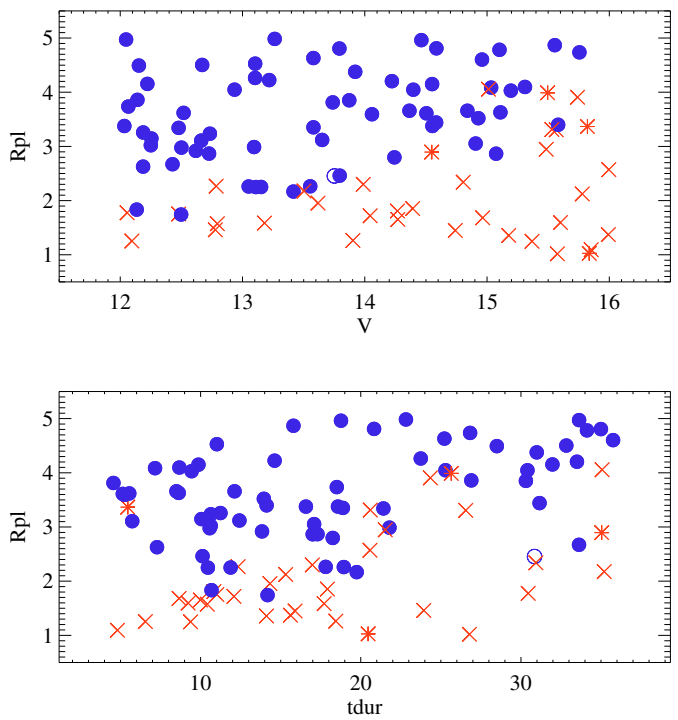

Fig. 10. Same as Figs. 7 and 8 but in the case of three $150 \mathrm{~d}$ observations separated by gaps of $150 \mathrm{~d}$.

detection algorithm, we prefiltered the data to removing low and high frequency noise.

Assuming a single observation of $150 \mathrm{~d}$, the period of at least 3 transits from a planet with a radius down to $\sim 2 R_{\oplus}$ can be recovered. The detection method does slightly depend on the apparent magnitude, but not on the transit duration in the given range of parameter values. The recovery of the right period does not depend on the number of transits, which only affects the confidence level (more transits will increase the confidence level).

We have also finally investigated the case of multiple observations $(3 \times 150 \mathrm{~d})$ of the same field, when regular gaps of $150 \mathrm{~d}$ are present and when one single transit is visible in all data sets.
In this case, assuming the period is close to $150 \mathrm{~d}$, the limit for the smallest detected planet does not differ from the case when all transits are observed in a single data set. These multiple observations may represent an application for the CoRoT or similar space missions to recover planetary transits when the orbital period is much longer than the single observation duration.

Acknowledgements. The availability of the VIRGO/SoHO data on total solar irradiance and spectral irradiances from the VIRGO Team through PMOD/WRC (Davos, Switzerland) and of unpublished data from the VIRGO Experiment on board the ESA/NASA Mission SoHO are gratefully acknowledged. We also gratefully acknowledge very constructive and instructive comments by $\mathrm{S}$. Aigrain and by H. Deeg.

\section{References}

Aigrain, S., \& Favata, F. 2002, A\&A, 395, 625

Aigrain, S., \& Irwin, M. 2004, MNRAS, 350, 331

Baglin, A. 2007, in prep.

Charbonneau, D., Brown, T. M., Latham, D. W., \& Mayor, M. 2000, ApJ, 529, L45

Carpano, S., Aigrain, S., \& Favata, F. 2003, A\&A, 401, 743

Deeg, H. 1999, http://www.iac.es/galeria/hdeeg/hdeeghome.html Fröhlich, C., Crommelynck, D. A., Wehrli, C., Anklin, M., et al. 1997, Sol. Phys., 175,267

Jenkins, J. M., Doyle, L. R., \& Cullers, D. K. 1996, Icarus, 119, 244

Kovács, G., Zucker, S., \& Mazeh, T. 2002, A\&A, 391, 369

Leahy, D. A., Darbro, W., Elsner, R. F., Weisskopf, M. C., et al. 1983, ApJ, 266, 160

Mayor, M., \& Queloz, D. 1995, Nature, 378, 355

Moutou, C., Pont, F., Barge, P., Aigrain, S., et al. 2005, A\&A, 437, 355

Protopapas, P., Jimenez, R., \& Alcock, C. 2005, MNRAS, 362, 460

Rauer, H., \& Eriksson, A. 2007, in Extrasolar Planets, ed. R. Dvorak (Weinheim: WILEY-VCH), 207

Tingley, B. 2003a, A\&A, 403, 329

Tingley, B. 2003b, A\&A, 408, L5

Udry S., Fischer, D., \& Queloz, D. 2007, in Protostars and Planets V, B. Reipurth, ed. D. Jewitt, \& K. Keil (Tucson: University of Arizona Press), 951, 915 